\title{
Resiliencia en los empleados con discapacidad de la Universidad Técnica de Manabí
}

\author{
Resilience in employees with disabilities at the Technical University of Manabí \\ Resiliência em funcionários com deficiência na Universidade Técnica de \\ Manabí
}

\author{
John Cristhian Velez Intriago \\ johncvelez@hotmail.com \\ https://orcid.org/0000-0002-8429-6157
}

Universidad Técnica de Manabí, Portoviejo, Ecuador
John Williams Velez Intriago

johnwvelezi@gmail.com

https://orcid.org/0000-0002-1465-8933

Universidad Técnica de Manabí, Portoviejo, Ecuador

Recibido: octubre 2020 / Revisado: noviembre 2020 / Aceptado: diciembre 2020 / Publicado: enero 2021

RESUMEN

La investigación tuvo como objetivo estudiar la capacidad resiliente en las personas discapacitadas que se desempeñan como empleados en la Universidad Técnica de Manabí. Se asistió del método inductivodeductivo para llegar a una conclusión general concreta sobre el tema, aplicando a una muestra poblacional de 24 personas la escala de Resiliencia SV-RES (Saavedra y Villalta, 2008) las tres Competencias interaccionales de Grotberg: Yo soy/yo estoy, yo tengo y yo puedo y la encuesta en línea. Se evidenció un nivel de resiliencia alto; a la vez, una minoría se encuentran indecisos y confundidos y otro grupo pequeño bajo. Se confirmó que las personas con discapacidad (visual, auditiva y/o motriz) logran un desempeño eficaz en determinados puestos de trabajo, siempre que se les garantice un adecuado nivel de vigilancia resiliente.

Palabras clave: Resiliencia; discapacidad; empleo; universidad
The investigation aimed study resilient capacity in disabled people who are perform as employees in the Technical University of Manabí. The inductive-deductive method was assisted to get to a concrete general conclusion about the subject, applying to a sample population of 24 people Resilience scale SV-RES (Saavedra and Villalta, 2008) the three Interactional competences from Grotberg: I am / I am, II have and I can and the survey online. A level was evidenced high resilience; at the same time, one minority are undecided and confused and another small group under. It was confirmed that people disabled (visual, auditory and / or motor) achieve a performance effective in certain positions of work, as long as they are ensure an adequate level of resilient surveillance.

Palabras clave: Resilience; disability; employment; university
RESUMO

A investigação teve como objetivo estudar capacidade resiliente em pessoas deficientes que são atuar como funcionários em a Universidade Técnica de Manabí. O método indutivo-dedutivo foi auxiliado para chegar a um conclusão geral concreta sobre - assunto, aplicando-se a uma amostra população de 24 pessoas Escala de resiliência SV-RES (Saavedra e Villalta, 2008) os três Competências interacionais de Grotberg: eu sou / sou, eu Eu tenho e posso e a pesquisa em linha. Um nível foi evidenciado alta resiliência; ao mesmo tempo, um minoria está indecisa e confuso e outro pequeno grupo debaixo. Foi confirmado que as pessoas desativado (visual, auditivo e / ou motor) atingir um desempenho eficaz em certas posições de trabalho, contanto que sejam garantir um nível adequado de vigilância resiliente.

Palavras-chave: Resiliência; deficiência; emprego; universidade 


\section{INTRODUCCIÓN}

Con la palabra discapacidad se resume un importante número de limitaciones funcionales que se registran en las poblaciones de todos los países del mundo. Puede revestir una deficiencia física, intelectual o sensorial, una dolencia que requiera atención médica o una enfermedad mental. Tales padecimientos son de carácter permanente o transitorio (Organización Mundial de la Salud, 2011).

Estimaciones recientes de dicha organización plantean que en el mundo alrededor del $15 \%$ de la población presenta esta condición, y en América Latina se ha reportado que aproximadamente el 10\% (Hurtado y Agudelo, 2014)

En un informe del Instituto Interamericano del Niño (OEA, 2015) sobre el cumplimiento del Programa de Acción Mundial para los Discapacitados de la ONU (1975), se plantea que "En el mundo de hoy hay un número grande y creciente de personas con incapacidades" (s.n.).

Se estima que por lo menos 350 millones de discapacitados viven en zonas donde no se dispone de los servicios necesarios para ayudarles a superar sus limitaciones. Los discapacitados están expuestos en gran medida a barreras físicas, culturales y sociales que obstaculizan su vida aun cuando se disponga de ayuda para la rehabilitación.

En el propio informe se enfatiza que en los países en desarrollo como Ecuador, hasta el $80 \%$ del total de los discapacitados viven en zonas rurales aisladas. El problema se agudiza cuando los discapacitados suelen ser sumamente pobres; a menudo viven en zonas donde los servicios médicos y afines son escasos o faltan totalmente, donde tampoco las incapacidades se descubren a tiempo. Cuando reciben atención médica, si la llegan a recibir, la deficiencia puede haberse hecho irreversible.

Relacionando esta calamidad con el derecho al trabajo en el propio informe se destaca que: en no pocos casos se niega otorgar empleo a muchas personas afectadas de discapacidad o sólo se les da trabajo servil y mal remunerado. Los discapacitados suelen ser los primeros a quienes se despide en épocas de desempleo y estrechez económica, a la vez suelen ser los últimos a los que se les contrata. Y ello es así a pesar de que se ha demostrado que con una adecuada evaluación, capacitación y colocación, la mayoría de los discapacitados pueden realizar eficientemente una amplia gama de tareas (OEA, 2015).

Cuando la economía familiar se basa en la agricultura y existe la tradicional familia extensa, se pueden dar tareas útiles a casi todos los discapacitados. Pero a medida que más familias se desplazan de las regiones rurales hacia los centros urbanos, que se produce la mecanización y comercialización de la agricultura, que las transacciones monetarias sustituyen al sistema de trueque y que la institución de la familia extensa se desintegra, se agudiza la falta de oportunidades de trabajo para los discapacitados. Los que viven en los barrios pobres de las ciudades enfrentan una fuerte competencia al buscar empleo y no hay muchas otras actividades económicamente productivas. Muchos de los discapacitados de estas zonas se ven forzados a la inacción y pasan a ser dependientes; otros han de recurrir a la mendicidad (OEA, 2015).

En este sentido y a pesar de las políticas inclusivas desplegadas por el estado ecuatoriano y de los esfuerzos realizados en la Universidad Técnica de Manabí, aún perduran en el ámbito laboral de la institución las reminiscencias de un pasado cercano, distinguido por el desprecio y la discriminación a las personas que padecen discapacidades.

Los discapacitados incorporados laboralmente continúan siendo blanco de discriminación, pues cuando menos se les observa como personas dignas de caridad humana que se les acepta laboralmente por puro compromiso social, sin 
comprender que estas personas no sólo necesitan trabajar por su condición humana, sino que también pueden realizar un aporte a la sociedad desde su desempeño digno en un puesto laboral.

La resiliencia juega un papel importante en estos casos ya que mediante su promoción, se puede favorecer el desarrollo de competencias laborales y personales, que permitan al empleado que padece una discapacidad sobreponerse a situaciones adversas y salir adelante en la vida.

Para efectos de comprensión conceptual conviene a estas alturas revisar lo concerniente a la resilencia y a la discapacidad.

El fenómeno que representa el término resiliencia no es nuevo, aunque lo parezca, su historia marcha junto a la tradición del hombre y se fundamenta en la creencia de que lo positivo siempre tiene que ser superior a lo negativo y que las fortalezas tendrán un mayor peso que las dificultades. Radica en la capacidad humana de afrontar las adversidades y salir fortalecidos de ellas. Para el criterio de algunos autores se manifiesta como una realidad que propone un modelo que enfatiza las fortalezas que los individuos son capaces de crear, condicionando su interacción con el medio a través de su paso por las distintas etapas vitales (Casas y Campos, 2015).

Discapacidad es un término general que abarca las deficiencias, las limitaciones de la actividad y las restricciones de la participación (OMS, 2011). Las deficiencias son problemas que afectan a una estructura o función corporal, las limitaciones de la actividad son dificultades para ejecutar acciones o tareas y las restricciones de la participación son desavenencias para existir en situaciones vitales. Por consiguiente, la discapacidad es un fenómeno complejo que refleja una interacción entre las características del organismo humano y las características de la sociedad en la que vive (OMS, 2011).

Considerando lo expuesto anteriormente la interrogante de la investigación consistió en
¿Cómo se comporta la capacidad resiliente de las personas con discapacidad de tipo visual, auditiva y motriz, que se desempeñan en diversos puestos de trabajo en la Universidad Técnica de Manabí? De acá se vinculan los objetivos siguientes:

\section{Objetivo general}

Estudiar la capacidad resiliente en las personas discapacitadas que se desempeñan como empleados en la Universidad Técnica de Manabí.

\section{Objetivos específicos}

1. Analizar la capacidad de resiliencia desde la propuesta de Saavedra (2008) en las personas discapacitadas que laboran en la Universidad Técnica de Manabí.

2. Conocer la evolución de la resiliencia a partir de las Competencias interaccionales de Grotberg (2005) en las personas discapacitadas que laboran en la Universidad Técnica de Manabí.

3. Evaluar el comportamiento de las variables resiliencia y discapacidad en la población mencionada anteriormente.

Se justifica este estudio debido a que el padecimiento de una discapacidad para una persona genera consecuencias desfavorables en todas las esferas de la vida (Rojas y Saucedo, 2014) La situación se agrava cuando no existe una adecuada aceptación y comprensión social sobre dicha problemática. Es por ello que la investigación presenta una importancia relevante, ya que contribuyó con la generación de conciencia por parte de los actores y la sociedad universitaria, sobre el proceso de inclusión social y laboral de las personas con discapacidad en la Universidad Técnica de Manabí.

En la búsqueda documental y bibliográfica realizada para la investigación, no se localizó algún trabajo investigativo que aborde la medición de la resiliencia en las personas con discapacidad de 
tipo visual, auditiva y motriz, que se desempeñan laboralmente en diversos puestos de trabajo en la Universidad Técnica de Manabí.

Con la investigación se favoreció el incremento de la conciencia en los actores y la sociedad universitaria, para que actúen en concordancia con lo establecido en la Ley de Discapacidades, y creen ambientes con las facilidades necesarias para que se realicen con normalidad, en un clima afectivo y de comprensión, las actividades laborales del personal que posee algún tipo de discapacidad, brindándoles respeto, cordialidad sin discriminación y la requerida igualdad de derechos.

\section{MÉTODO}

Se empleó el método inductivo-deductivo como estrategia de razonamiento lógico, utilizando premisas particulares para llegar a una conclusión general concreta sobre el tema que se estudia, usando los principios generales para llegar a conclusiones específicas.

A una muestra poblacional de 24 personas se puso en práctica la técnica de la observación directa y la indirecta, así como la encuesta en línea aprovechando los medios telemáticos disponibles para la aplicación de la escala de Resiliencia SVRES (Saavedra y Villalta, 2008) constituida por 60 ítemes para analizar el comportamiento de las tres Competencias interaccionales de Grotberg: Yo soy/ yo estoy, yo tengo y yo puedo (2005)

Se contó con un formato tipo Lickert que se califica con un rango de 5 opciones de respuesta que representan los niveles de estructuración de la conciencia: (1) Muy de acuerdo (2) De acuerdo (3) $\mathrm{Ni}$ acuerdo ni desacuerdo (4) En desacuerdo y (5) Muy en desacuerdo.

\section{RESULTADOS}

A manera de ejemplo y para efectos operativos de la comprención metodológica y del análisis de la información, en adelante se presentan una serie de cuadros y de gráficos que de alguna manera representan el universo poblacional y los objetivos que propulsan la investigación.

En la Figura 1 se muestra el tipo de discapacidad que padecen los 24 trabajadores de la Universidad Técnica de Manabí.

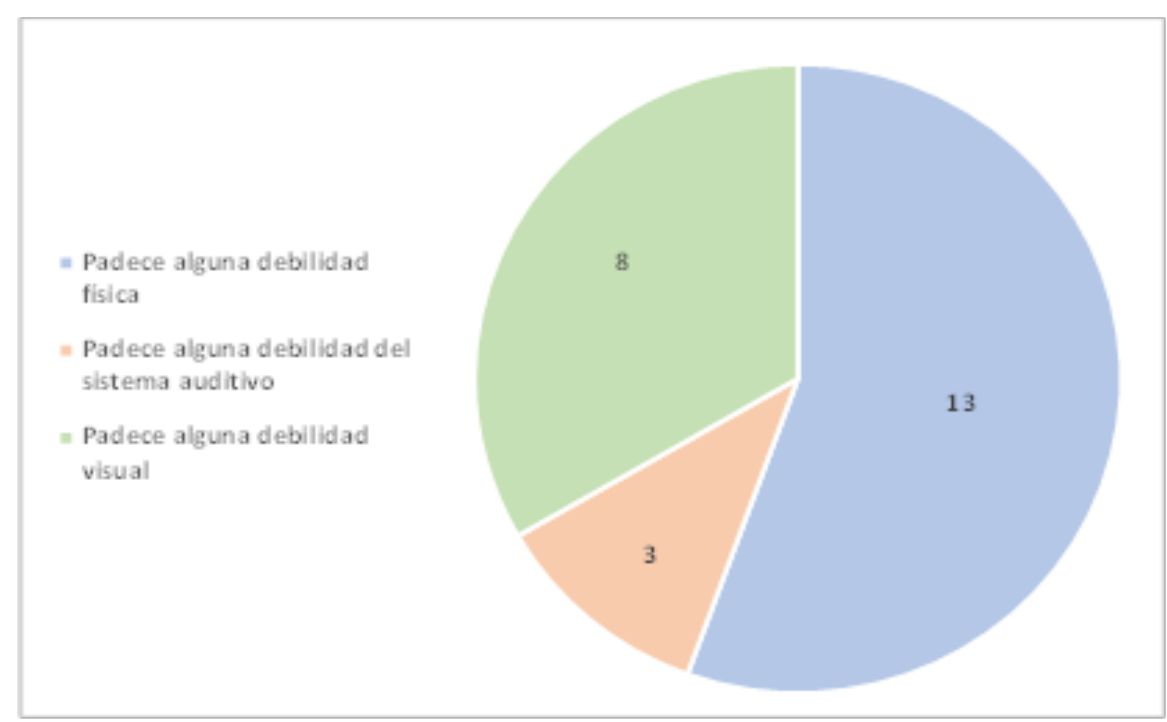

Figura 1. Distribución gráfica de las discapacidades. (Fuente: elaboración propia a partir de los datos de la encuesta). 
Se apreció en los resultados que las discapacidades que predominan en las trabajadoras y en los trabajadores de la UTM, corresponden a debilidades físicas y dificultades auditivas. La minoría confronta debilidad visual.

\section{Resultados de la aplicación de la escala SV-RES}

En la Tabla 1 se muestran los resultados de la aplicación de la escala de resiliencia en función de la verbalización de Grotberg: Yo Soy/Yo Estoy, según el nivel de estructuración de la conciencia.

Tabla 1. Resultado estadístico de las respuestas en la verbalización: Yo Soy/Yo Estoy, según niveles de estructuración de la conciencia.

\begin{tabular}{|c|c|c|c|c|c|c|}
\hline No & $\begin{array}{l}\text { Itens de la Competencia interaccional } \\
\text { Yo Soy/Yo Eestoy }\end{array}$ & $\begin{array}{l}\text { Muy de } \\
\text { acuerdo }\end{array}$ & $\begin{array}{c}\text { De } \\
\text { acuerdo }\end{array}$ & $\begin{array}{l}\text { Ni acuerdo } \\
\text { ni en } \\
\text { desacuerdo }\end{array}$ & $\begin{array}{c}\text { En } \\
\text { desacuerdo }\end{array}$ & $\begin{array}{c}\text { Muy en } \\
\text { desacuerdo }\end{array}$ \\
\hline 1 & Yo soy una persona con esperanza & 13 & 6 & 5 & 0 & 0 \\
\hline 2 & Yo soy una persona con buena autoestima & 13 & 5 & 3 & 3 & 0 \\
\hline 3 & Yo soy optimista respecto al futuro & 11 & 10 & 3 & 0 & 0 \\
\hline 4 & Yo estoy seguro de mis creencias o principios & 11 & 10 & 3 & 0 & 0 \\
\hline 5 & Yo estoy creciendo como persona & 10 & 11 & 3 & 0 & 0 \\
\hline 6 & $\begin{array}{l}\text { Yo estoy rodeado de personas que, en general, me } \\
\text { ayudan en situaciones difíciles }\end{array}$ & 11 & 8 & 5 & 0 & 0 \\
\hline 7 & Yo estoy en contacto con personas que me aprecian & 11 & 8 & 5 & 0 & 0 \\
\hline 8 & Yo estoy seguro de mí mismo & 13 & 8 & 3 & 0 & 0 \\
\hline 9 & Yo estoy seguro de mis proyectos y metas & 10 & 11 & 3 & 0 & 0 \\
\hline 10 & Yo estoy seguro en el ambiente en el que vivo & 5 & 14 & 5 & 0 & 0 \\
\hline 11 & $\begin{array}{l}\text { Yo soy una persona que ha aprendido a salir adelante } \\
\text { en la vida }\end{array}$ & 10 & 11 & 3 & 0 & 0 \\
\hline 12 & Yo soy un modelo positivo para otras personas & 8 & 13 & 3 & 0 & 0 \\
\hline 13 & Yo estoy bien integrado en mi lugar de trabajo o estudio & 5 & 11 & 8 & 0 & 0 \\
\hline 14 & Yo estoy satisfecho con mis relaciones de amistad & 8 & 8 & 8 & 0 & 0 \\
\hline 15 & Yo estoy satisfecho con mis relaciones afectivas & 5 & 11 & 5 & 3 & 0 \\
\hline 16 & Yo soy una persona práctica & 11 & 8 & 2 & 3 & 0 \\
\hline 17 & Yo soy una persona con metas en la vida & 8 & 8 & 8 & 0 & 0 \\
\hline 18 & Yo estoy activo frente a mis problemas & 8 & 8 & 5 & 3 & 0 \\
\hline 19 & Yo estoy revisando constantemente el sentido de la vida & 8 & 11 & 5 & 0 & 0 \\
\hline 20 & Yo estoy generando soluciones a mis problemas & 8 & 11 & 5 & 0 & 0 \\
\hline
\end{tabular}

Fuente: elaboración propia a partir de los datos de la encuesta. 
Se apreció que el $79 \%$ de los encuestados respondieron entre muy de acuerdo y de acuerdo; el $19 \%$ se encuentran indecisos y el $2 \%$ considera estar en desacuerdo.

Los items que demostraron estar de acuerdo corresponden a una persona con esperanzas, tener una buena autoestima, ser optimistas respecto al futuro, estar seguro de sus creencias y principios, el estar creciendo como persona, el estar rodeado de personas que le ayudan en situaciones difíciles, estar en contacto con personas que le aprecian, estar seguro de sí mismo, estar seguro de sus metas y proyectos, a estar seguro en el ambiente que vive, ser una persona que ha aprendido a salir adelante en la vida, ser un modelo positivo para otras personas, estar integrado en el lugar de trabajo, la satisfacción con las relaciones de amistad, la satisfacción con las relaciones afectivas, ser una persona práctica, ser una persona con metas en la vida, estar activo ante los problemas, estar revisando constantemente el sentido de la vida y estar generando soluciones a sus problemas.

La situación crítica se produce en los resultados que demuestran desacuerdo, donde se apreció que existe una minoría que manifestó lo siguiente: no ser una persona con buena autoestima, no encontrarse satisfecho con sus relaciones afectivas, no ser una persona práctica y no encontrarse activo frente a los problemas.

En la Tabla 2 se mostraron los resultados de la aplicación de la escala de resiliencia en función de la verbalización de Grotberg: Yo Tengo, según el nivel de estructuración de la conciencia.

Tabla 2. Resultado estadístico de las respuestas en la verbalización: Yo Tengo, según niveles de estructuración de la conciencia.

\begin{tabular}{|c|c|c|c|c|c|c|}
\hline No & $\begin{array}{c}\text { Itens de la Competencia interaccional } \\
\text { Yo Tengo }\end{array}$ & $\begin{array}{l}\text { Muy de } \\
\text { acuerdo }\end{array}$ & $\begin{array}{c}\text { De } \\
\text { acuerdo }\end{array}$ & $\begin{array}{l}\text { Ni acuerdo } \\
\text { ni en } \\
\text { desacuerdo }\end{array}$ & $\begin{array}{c}\text { En } \\
\text { desacuerdo }\end{array}$ & $\begin{array}{c}\text { Muy en } \\
\text { desacuerdo }\end{array}$ \\
\hline 1 & Yo tengo relaciones personales confiables & 11 & 5 & 8 & 0 & 0 \\
\hline 2 & Yo tengo una familia bien estructurada & 5 & 16 & 3 & 0 & 0 \\
\hline 3 & Yo tengo relaciones afectivas sólidas & 8 & 11 & 5 & 0 & 0 \\
\hline 4 & Yo tengo fortaleza anterior & 8 & 13 & 3 & 0 & 0 \\
\hline 5 & Yo tengo una vida con sentido & 11 & 10 & 3 & 0 & 0 \\
\hline 6 & Yo tengo acceso a servicios públicos & 13 & 6 & 5 & 0 & 0 \\
\hline 7 & Yo tengo personas que me apoyan & 11 & 8 & 5 & 0 & 0 \\
\hline 8 & Yo tengo a quién recurrir en caso de problemas & 8 & 11 & 2 & 3 & 0 \\
\hline 9 & $\begin{array}{l}\text { Yo tengo personas que estimulan mi autonomía e } \\
\text { iniciativa }\end{array}$ & 5 & 13 & 3 & 3 & 0 \\
\hline 10 & $\begin{array}{l}\text { Yo tengo satisfacción con lo que he logrado en la } \\
\text { vida }\end{array}$ & 5 & 11 & 5 & 3 & 0 \\
\hline 11 & $\begin{array}{l}\text { Yo tengo personas que me han orientado y } \\
\text { aconsejado }\end{array}$ & 5 & 14 & 5 & 0 & 0 \\
\hline
\end{tabular}




\begin{tabular}{|c|c|c|c|c|c|c|}
\hline No & $\begin{array}{l}\text { Itens de la Competencia interaccional } \\
\text { Yo Soy/Yo Eestoy }\end{array}$ & $\begin{array}{l}\text { Muy de } \\
\text { acuerdo }\end{array}$ & $\begin{array}{l}\text { De } \\
\text { acuerdo }\end{array}$ & $\begin{array}{c}\text { Ni acuerdo } \\
\text { ni en } \\
\text { desacuerdo }\end{array}$ & $\begin{array}{c}\text { En } \\
\text { desacuerdo }\end{array}$ & $\begin{array}{c}\text { Muy en } \\
\text { desacuerdo }\end{array}$ \\
\hline 12 & $\begin{array}{l}\text { Yo tengo personas que me ayudan a evitar peligros o } \\
\text { problemas }\end{array}$ & 8 & 8 & 8 & 0 & 0 \\
\hline 13 & Yo tengo personas en las cuales puedo confiar & 8 & 11 & 5 & 0 & 0 \\
\hline 14 & $\begin{array}{l}\text { Yo tengo personas que han confiado sus problemas en } \\
\mathrm{mi}\end{array}$ & 11 & 10 & 3 & 0 & 0 \\
\hline 15 & $\begin{array}{l}\text { Yo tengo personas que me han acompañado cuando he } \\
\text { tenido problemas }\end{array}$ & 5 & 16 & 3 & 0 & 0 \\
\hline 16 & Yo tengo metas a corto plazo & 5 & 14 & 5 & 0 & 0 \\
\hline 17 & Yo tengo mis objetivos claros & 8 & 11 & 5 & 0 & 0 \\
\hline 18 & Yo tengo personas con quien enfrentar los problemas & 11 & 10 & 3 & 0 & 0 \\
\hline 19 & Yo tengo proyectos a futuro & 8 & 11 & 5 & 0 & 0 \\
\hline 20 & Yo tengo problemas que puedo solucionar & 5 & 14 & 5 & 0 & 0 \\
\hline
\end{tabular}

Fuente: elaboración propia a partir de los datos de la encuesta.

La información estadística mostrada anteriormente precisó que el $79 \%$ de los encuestados respondieron entre muy de acuerdo y de acuerdo; el $19 \%$ se encuentran indecisos y el $2 \%$ consideró estar en desacuerdo.

Los items que demostraron estar de acuerdo corresponden a tener relaciones personales confiables, tener una familia bien estructurada, sostener relaciones afectivas sólidas, tener fortaleza interior, sostener una vida con sentido, tener acceso a servicios públicos, tener personas que lo apoyan, tener a quién recurrir en caso de problemas, contar con personas que estimulan su autonomía e iniciativa tener satisfacción con lo que ha logrado en la vida, contar con personas que lo han orientado y aconsejado, tener personas que lo ayudan a evitar peligros o problemas, contar con personas en las cuales puede confiar, tener personas que han confiado sus problemas en él, tener personas que lo han acompañado cuando ha tenido problemas, tener metas a corto plazo, tener sus objetivos claros, contar con personas con quien enfrentar los problemas, tener proyectos a futuro y tener problemas que puede solucionar.

La situación más crítica se produce en los resultados que demuestran desacuerdo, donde se puede apreciar que existe una minoría que manifiesta lo siguiente: no tener a quién recurrir en caso de problemas, no tener personas que estimulen su autonomía e iniciativa y no tener satisfacción con lo que ha logrado en la vida.

En la Tabla 3 se mostraron los resultados de la aplicación de la escala de resiliencia en función de la verbalización de Grotberg: Yo Puedo, según el nivel de estructuración de la conciencia. 
Tabla 3. Resultado estadístico de las respuestas en la verbalización: Yo Puedo, según niveles de estructuración de la conciencia.

\begin{tabular}{|c|c|c|c|c|c|c|}
\hline No & $\begin{array}{l}\text { Itens de la Competencia interaccional } \\
\text { Yo Tengo }\end{array}$ & $\begin{array}{l}\text { Muy de } \\
\text { acuerdo }\end{array}$ & $\begin{array}{c}\text { De } \\
\text { acuerdo }\end{array}$ & $\begin{array}{c}\text { Ni acuerdo } \\
\text { ni en } \\
\text { desacuerdo }\end{array}$ & $\begin{array}{c}\text { En } \\
\text { desacuerdo }\end{array}$ & $\begin{array}{c}\text { Muy en } \\
\text { desacuerdo }\end{array}$ \\
\hline 1 & Yo puedo hablar de mis emociones & 8 & 13 & 3 & 0 & 0 \\
\hline 2 & Yo puedo expresar afecto & 13 & 8 & 3 & 0 & 0 \\
\hline 3 & Yo puedo confiar en las personas & 8 & 13 & 3 & 0 & 0 \\
\hline 4 & $\begin{array}{l}\text { Yo puedo superar las dificultades que se me } \\
\text { presentan en la vida }\end{array}$ & 5 & 11 & 8 & 0 & 0 \\
\hline 5 & Yo puedo desarrollar vínculos afectivos & 5 & 13 & 3 & 3 & 0 \\
\hline 6 & Yo puedo resolver problemas de manera afectiva & 8 & 13 & 3 & 0 & 0 \\
\hline 7 & Yo puedo dar mi opinión & 5 & 13 & 5 & 0 & 0 \\
\hline 8 & Yo puedo buscar ayuda cuando la necesito & 8 & 11 & 5 & 0 & 0 \\
\hline 9 & Yo puedo apoyar a otros que tienen dificultades & 11 & 11 & 3 & 0 & 0 \\
\hline 10 & Yo puedo responsabilizarme por lo que hago & 11 & 8 & 5 & 0 & 0 \\
\hline 11 & Yo puedo ser creativo & 8 & 11 & 5 & 0 & 0 \\
\hline 12 & Yo puedo comunicarme adecuadamente & 8 & 13 & 3 & 0 & 0 \\
\hline 13 & Yo puedo aprender de mis aciertos y mis errores & 11 & 8 & 5 & 0 & 0 \\
\hline 14 & $\begin{array}{l}\text { Yo puedo colaborar con otros para mejorar la vida } \\
\text { en la comunidad }\end{array}$ & 11 & 8 & 5 & 0 & 0 \\
\hline 15 & Yo puedo tomar decisiones & 11 & 10 & 3 & 0 & 0 \\
\hline 16 & $\begin{array}{l}\text { Yo puedo generar estrategias para solucionar mis } \\
\text { problemas }\end{array}$ & 8 & 11 & 5 & 0 & 0 \\
\hline 17 & Yo puedo fijarme metas realistas & 5 & 16 & 3 & 0 & 0 \\
\hline 18 & Yo puedo esforzarme por lograr mis objetivos & 5 & 16 & 3 & 0 & 0 \\
\hline 19 & Yo puedo asumir riesgos & 8 & 11 & 5 & 0 & 0 \\
\hline 20 & Yo puedo proyectarme al futuro & 11 & 10 & 3 & 0 & 0 \\
\hline
\end{tabular}

Fuente: elaboración propia a partir de los datos de la encuesta. 
La información estadística precisó que el $83 \%$ de los encuestados respondieron entre muy de acuerdo y de acuerdo; el $16 \%$ se encuentran indecisos y el $1 \%$ considera estar en desacuerdo.

Los items que demostraron estar de acuerdo corresponden a personas que pueden hablar de sus emociones, poder expresar afecto, poder confiar en las personas, poder superar las dificultades que se le presentan en la vida, poder desarrollar vínculos afectivos, poder resolver problemas de manera afectiva, poder dar su opinión, poder buscar ayuda cuando la necesite, poder apoyar a otros que tienen dificultades, poder responsabilizarse por lo que haga, poder ser creativo, poder comunicarse adecuadamente, poder aprender de sus aciertos y errores, poder colaborar con otros para mejorar la vida en la comunidad, estar en condiciones de poder tomar decisiones, poder generar estrategias para solucionar sus problemas, poder fijarse metas realistas, estar en condiciones de esforzarse por lograr sus objetivos, poder asumir riesgos y poder proyectarse al futuro.

La situación menos ventajosa se produce con tres participantes que manifestaron no poder desarrollar vínculos afectivos.

En los resultados mostrados anteriormente se apreció que en todos los casos el nivel de resiliencia muestra una calificación alta, sin embargo, cuando se realizó un análisis general de la información se advirtió que existe un balance más o menos equilibrado entre los tres niveles de estructuración de la conciencia, con excepción los que se mostraron indecisos y los que están en desacuerdo.

\section{Reporte de los resultados}

Cuando se analizaron los datos reflejados en las Tablas 5, 6 y 7, relacionado con el análisis de los resultados de la encuesta en las Competencias interacciónales de Grotberg, se precisó que en todos los ítems hubo un nivel de inseguridad e indecisión por parte de algunos encuestados. Dicha situación aunque no resulta crítica, se debe constituir en una preocupación para los especialistas del trabajo psicosocial, pues de continuar empeorando, puede afectar sensiblemente el nivel de resiliencia de las personas y el colectivo.

La investigación permitió verificar que la mayoría de las respuestas de los encuestados se enmarcan en los niveles de estructuración de la conciencia Muy de Acuerdo y De Acuerdo, demostrando que los encuestados son capaces de definirse como sujetos relativamente estables, que donde desempeñan sus actividades laborales han podido interpretar sus funciones y cumplirlas con calidad, como personas que les gusta ayudar y que son capaces de demostrar afectos por sus compañeros, expresando empatía basado en el ejemplo práctico que se sustenta en metas concretas para la vida.

Al mismo tiempo se apreció que la mayoría de los encuestados son capaces de establecer juicios, que ponen de relieve el valor del colectivo laboral como red social de importancia, que tienen sus raíces en la historia personal, confirmando que son capaces de establecer relaciones personales confiables, y relaciones afectivas sólidas. Manifiestan tener personas que lo apoyan y estimulan su autonomía e iniciativa y con las que pueden contar para que les ayuden ante cualquier problema. Plantean tener objetivos claros y poder contar con ellos para enfrentar los problemas y proyectos del futuro.

También se observó que la mayoría de los encuestados son capaces de establecer juicios de valor que se refieren a las potencialidades que poseen para establecer vínculos con el entorno laboral y social, manteniendo buen humor y relaciones de confianza con el colectivo. Son capaces de resolver problemas de manera efectiva, con posibilidad de reconocer y corregir los errores, pudiendo ayudar a sus compañeros de trabajo 
que tienen dificultades, demostrando creatividad, persistencia y poder crear experiencias positivas a partir de los aciertos y errores. Plantearon ser capaces de trazar estrategias para solucionar los problemas, esforzarse para lograr los objetivos propuestos y trabajar en conjunto con los miembros del colectivo laboral en la solución de nuevos problemas.

Al mismo tiempo se verificó que una minoría de las personas encuestadas manifestaron estar confundidos e indecisos, que no son capaces de definir una postura resiliente de manera consciente, que poseen dudas para establecer juicios de conciencia en base a sus capacidades y se muestran confundidos, con dudas de sus capacidades, no logrando reconocer la posibilidad de tener éxitos por sí mismos y no se sienten capacitados para pedir ayuda.

La situación más crítica se presenta en un grupo bien reducido de las personas encuestadas que requieren una atención urgente, pues se mostraron como personas no resilientes, no logrando identificarse como tales, con poca autonomía y satisfacción por sus actos, sin metas a futuro, no pudiendo concluir los proyectos de vida que logran emprender y sin poder establecer relaciones personales confiables.

Otros problemas relacionados con este pequeño grupo de personas, es que consideraron que el colectivo laboral no se encuentra sólidamente estructurado, ni reconocen el apoyo de otras personas, sintiéndose inconformes con lo que han logrado en la vida, no pudiendo trazarse objetivos claros ni establecer proyectos a futuro, se mantienen aislados sin compartir sus emociones con otros miembros del colectivo laboral, encontrándose generalmente de mal humor, no logrando resolver problemas de manera efectiva, ni se sienten con capacidad de apoyar a los miembros del colectivo que tienen dificultades, no siendo capaces de aprender de sus errores ni de trazar estrategias para solucionar sus problemas.

Lo analizado anteriormente confirmó que las personas con discapacidad (visual, auditiva y/o motriz) logran un desempeño eficaz en determinados puestos de trabajo, siempre que se les garantice un adecuado nivel de vigilancia resiliente.

\section{CONCLUSIONES}

Se comprobó la relación estrecha que existe entre la resiliencia y las Competencias interacciónales de Grotberg, con los niveles de estructuración de la conciencia y la verbalización yo soy/yo estoy, yo tengo y yo puedo.

El trabajo evidenció un nivel de resiliencia alto en las personas discapacitadas que se desempeñan como empleados de la Universidad Técnica de Manabí. Demostrando que son capaces de definirse como sujetos relativamente estables, que donde desempeñan sus actividades laborales han interpretado sus funciones y las han cumplido a calidad; colaborando y demostrando afectos por sus compañeros, expresando empatía basado en el ejemplo práctico que se sustenta en metas concretas para la vida. Así mismo existe una minoría que se encuentran indecisos y confundidos y un grupo pequeño que presenta una resiliencia baja.

En los indecisos y lo que están en desacuerdo se destaca un nivel de inseguridad que aunque no resulta crítica, debe constituir una preocupación para los operadores del trabajo psicosocial de la empresa. Entre un $15 \%$ y un $18 \%$ de la muestra estudiada manifestaron no ser capaces de definir una postura resiliente de manera consciente, que poseen dudas para establecer juicios de conciencia en base a sus capacidades, no logrando reconocer la posibilidad de tener éxitos por sí mismos ni sintiéndose capacitados para pedir ayuda. 
Se confirmó que las personas con discapacidad (visual, auditiva y/o motriz) logran un desempeño eficaz en determinados puestos de trabajo, siempre que se les garantice un adecuado nivel de vigilancia resiliente.

\section{REFERENCIAS}

Casas, G., y Campos, I. (2015). Enfoque de la Resiliencia en el Trabajo Social. Facultad de Ciencias Sociales, Escuela de Trabajo Social, Universidad de Costa Rica, Consultado el 16 de agosto de 2020. www.ts.ucr.ac.cr

Grotberg, E. (2005). Nuevas tendencias en resiliencia. En A. Melillo y E. Suárez (Comps.) Resiliencia. Descubriendo las propias fortalezas (1930). Buenos Aires: Paidós

Hurtado, T., Y Agudelo, A. (2014). Inclusión educativa de las personas con discapacidad en Colombia. Revista CES Movimiento y Salud. 2014; v 2(1) : 45-55, Vol. 2 - No. 1

OEA. (1999). Convención Interamericana para la eliminación de todas las formas de discriminación contra las personas con discapacidad. Organización de Estados Americanos. Repositorio.dpe.gob.ec/ handle/39000/611

OEA. (2015). Informe sobre el Programa de Acción Mundial para los Discapacitados. Instituto Interamericano del Niño, Organismo Especializadode la Organización de Estados
Americanos (OEA), p.1. Recuperado de: http://www.iin.oas.org/Cursos_a_distancia/ Lectura\%201_disc.UT1.pdf

OMS. (1993). Normas Uniformes de las Naciones Unidas sobre la igualdad de oportunidades para las personas con discapacidad. Organización Mundial de la Salud de Las Naciones Unidas. Resolución aprobada por la Asamblea General [sobre la base del informe de la Tercera Comisión (A/48/627)]. https:// www.ohchr.org/SP/ProfessionalInterest/Pages/ PersonsWithDisabilities.aspx

OMS.(2011). Informe mundial sobre la discapacidad. Organización Mundial de la Salud, Ginebra, Suiza, 388

ONU. (1975). Declaración de los derechos de los discapacitados, de 9 de diciembre de 1975, adoptada por la Asamblea General de las Naciones Unidas, en su resolución A/ RES/3447(XXX). Asamblea General de las Naciones Unidas. Resolución A/RES/3447(XXX). (9 de diciembre de 1975). https://www. bienestaryproteccioninfantil.es/fuentes1. asp? sec $=13$ \&subs $=16$ \&cod $=633$ \&page $=$

Rojas, S. y Saucedo, A. (2014). Oftalmología. 1era edición. México: Editorial El Manual Moderno

Saavedra, y Villalta. (2008). Medición de las características resilientes, un estudio comparativo en personas entre 15 y 65 años. LIBERABIT, v 14:31-40. 2008. Lima, Perú. Universidad Católica de Maule. Chile, ISSN: 1729-4827 